\title{
Critical scaling for yield is independent of distance to isostaticity
}

\author{
Jacob D. Thompson (i) and Abram H. Clark \\ Department of Physics, Naval Postgraduate School, Monterey, California 93943, USA
}

(Received 26 March 2019; revised manuscript received 14 May 2019; published 9 August 2019)

\begin{abstract}
Using discrete element simulations, we demonstrate that critical behavior for yielding in soft disk and sphere packings is independent of the distance to isostaticity over a wide range of dimensionless pressures. Jammed states are explored via quasistatic shear at fixed pressure, and the statistics of the dimensionless shear stress $\mu$ of these states obey a scaling description with a diverging length scale $\xi \propto\left|\mu-\mu_{c}\right|^{-\nu}$. The critical scaling functions and values of the scaling exponents are nearly independent of distance to isostaticity despite the large range of pressures studied. Our results demonstrate that yielding of jammed systems represents a distinct nonequilibrium critical transition from the isostatic critical transition which has been demonstrated by previous studies. Our results may also be useful in deriving nonlocal rheological descriptions of granular materials, foams, emulsions, and other soft particulate materials.
\end{abstract}

DOI: 10.1103/PhysRevResearch.1.012002

Granular materials, dense suspensions, foams, and emulsions can form amorphous jammed states [1-5]. Jammed systems can yield when subjected to a sufficient shear stress $\tau$ (this is sometimes called "unjamming by shear"). When $\mu \equiv \tau / p$, where $p$ is the system pressure, exceeds a critical value $\mu_{c}$, jammed states become inaccessible and flow persists indefinitely [6-13]. Predicting the mechanical response of jammed states can be difficult since it can involve plastic rearrangement events that cooperate over large distances. For example, rheological models of these materials that include nonlocal cooperative effects can successfully reproduce steady-state flows from experiments and particle-based simulations [14-21].

Prior studies on soft sphere packings, which are commonly used to model these materials, have framed long-range cooperative behavior in terms of a nonequilibrium critical transition that occurs at the isostatic point, also called "point $J$ " [2,22-28]. Isostaticity refers to the number of contacts per particle $Z$ being equal to the number required to constrain all degrees of freedom in the system, $Z=Z_{\text {iso. This occurs at a }}$ given volume fraction $\phi=\phi_{c}$ in the large-system limit. At isostaticity, $p=0$, but further compression leads to increasing $p$. A cooperative length scale $\xi_{J} \propto\left|\phi-\phi_{J}\right|^{-v_{J}}$ diverges at the isostatic point, which then controls the mechanical response and leads to Widom-like scaling relations [29] that relate $p$, $\left(\phi-\phi_{J}\right),\left(Z-Z_{\text {iso }}\right)$, and other quantities. $\xi_{J}$ is large near isostaticity (i.e., small $p$ ), characterized by an excess of spatially extended, low-energy modes of the system [30,31]. For increasing $p, \xi_{J}$ decreases, leading to more localized modes as well as smaller and more localized particle rearrangements.

In contrast, nonlocal rheological descriptions of jammed materials [16,18-21,32] often include a diverging

Published by the American Physical Society under the terms of the Creative Commons Attribution 4.0 International license. Further distribution of this work must maintain attribution to the author(s) and the published article's title, journal citation, and DOI. cooperative length scale that depends not on packing fraction but on distance to a critical shear stress, i.e., $\xi \propto\left|\mu-\mu_{c}\right|^{-v}$. These rheological models, including our previous paper [32], describe materials that are near $\phi=\phi_{J}$, so it is not known how the cooperative length scale underlying these models relates to the isostatic critical point. Here, we show using numerical simulations that yielding in soft sphere packings is a distinct nonequilibrium critical transition and that it is independent of the distance to isostaticity. We quasistatically shear systems of repulsive, bidisperse disks and spheres, holding dimensionless pressure $\tilde{p}$ fixed and measuring $\mu$, which increases during an initial shear regime and then plateaus as stress is released in intermittent slips. The statistics of $\mu$ obey a scaling description with a diverging length scale $\xi \propto\left|\mu-\mu_{c}\right|^{-v}$, where $v_{\mathrm{ms}} \approx 1.8$ during initial shear buildup (in agreement with Ref. [32]) and $v_{\text {slip }} \approx 1.1$ in two dimensions $(2 \mathrm{D})$ and $v_{\text {slip }} \approx 0.8$ in three dimensions $(3 \mathrm{D})$ during the intermittent slip regime. The scaling functions and the values of $v$ are highly insensitive to the distance from isostaticity set by $\tilde{p}$, which we vary over nearly four orders of magnitude. $\mu_{c}(\tilde{p})$ is insensitive to $\tilde{p}$ for $\tilde{p} \leqslant 10^{-3}$, but decreases logarithmically for higher $\tilde{p}$. The critical scaling functions we show could be used to derive a particle-scale theory for nonlocal rheological models, including transient behavior, which is not captured by current models.

Methods. We use molecular dynamics simulations to study systems of $N$ bidisperse frictionless disks in 2D and spheres in $3 \mathrm{D}$, with a diameter ratio 1.4 and equal numbers of each size. Systems are prepared at a given pressure $p$ via isotropic compression and then quasistatically sheared. Contacting particles interact via a purely repulsive force $\mathbf{F}_{i j}=K\left(\delta_{i j} /\left|\mathbf{r}_{i j}\right|-1\right) \mathbf{r}_{i j}$, where $\delta_{i j}$ is the average diameter of particles $i$ and $j$, and $\mathbf{r}_{i j}$ is the vector displacement between the centers of particles $i$ and $j$. Stresses are quantified by the Cauchy stress tensor,

$$
\sigma_{\alpha \lambda}=\frac{1}{V} \sum_{i \neq j} r_{\alpha}^{i j} F_{\lambda}^{i j}
$$


(a)

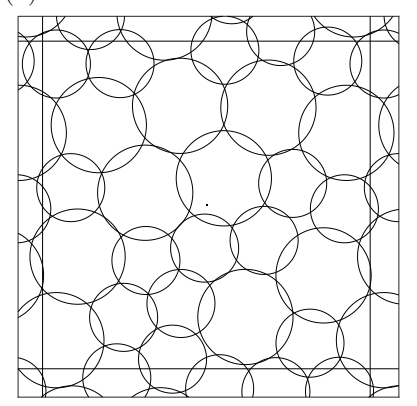

(b)

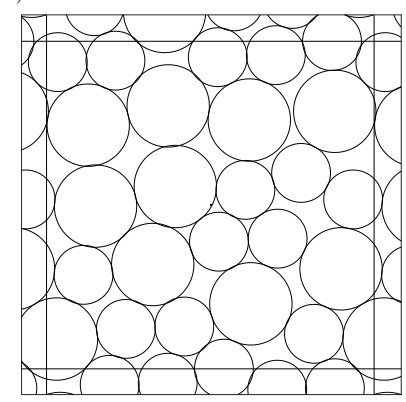

(c)

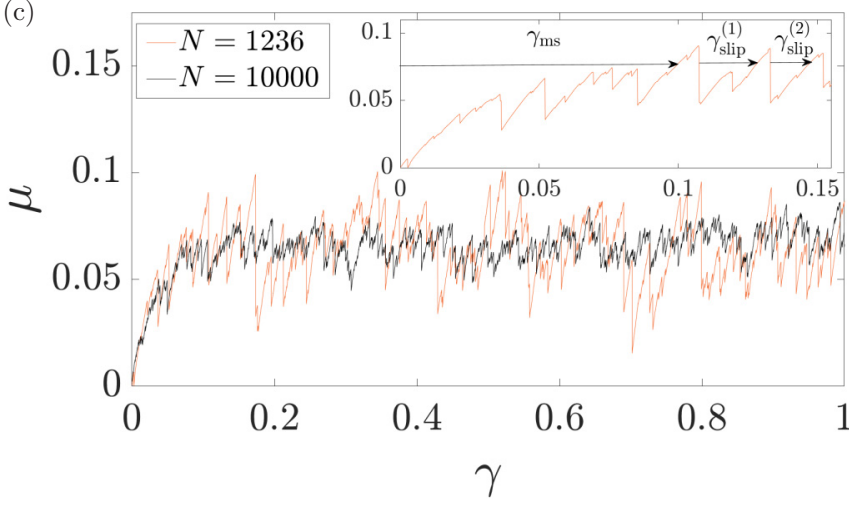

FIG. 1. (a), (b) Illustrative snapshots during Lees-Edwards shear at $\gamma=0.33$ with dimensionless pressure (a) $\tilde{p} \equiv p / K=0.2$ (far from isostaticity) and (b) $\tilde{p}=0.001$ (near isostaticity). (c) Plot of dimensionless shear stress $\mu$ vs $\gamma$ for a single simulation with 1236 (orange) and 10000 (black) particles. The inset shows a closeup of $0 \leqslant \gamma \leqslant 0.15$. The first arrow indicates the initial shear strain $\gamma_{\mathrm{ms}}$ required to find the first state at a particular value of $\mu$ (in the case shown, $\mu \approx 0.077$ ). Subsequent arrows denote the shear strains $\gamma_{\text {slip }}$ between states where the shear stress is less than the value of $\mu$ being considered.

Here, $\alpha$ and $\lambda$ are Cartesian coordinates, $V$ is the system volume, $r_{\alpha}^{i j}$ is the $\alpha$ component of the center-to-center separation vector between particles $i$ and $j$, and $F_{\lambda}^{i j}$ is the $\lambda$ component of the interparticle contact force. The sum over $i$ and $j$ includes all pairs of contacting particles.

Each simulation is prepared by placing particles randomly throughout the domain and then increasing the particle diameter $D$ in small steps until reaching a target $p=\left(\sigma_{x x}+\sigma_{y y}\right) / 2$. Using Lees-Edwards boundary conditions, we impose affine shear strain in small steps $\Delta \gamma=10^{-4}$. At each shear step, the shear-periodic boundary is moved by $\Delta \gamma$ and $y \Delta \gamma$ is added to the $x$ position of each particle. We then use molecular dynamics to relax the potential energy using modified velocity Verlet integration, as well as shrink or swell $D$ to maintain a fixed $p$ within $0.5 \%$ of the target value. Before shearing or changing the particle diameter, we damp out kinetic energy via a viscous damping force $-B \mathbf{v}$ to each particle, where $\mathbf{v}$ is the absolute velocity of a given particle and $B$ is the damping coefficient. We set $B=5 \sqrt{M p}$ in $2 \mathrm{D}$ and $B=5 \sqrt{M p D}$ in $3 \mathrm{D}$, where $M$ is the mass of a large grain. Our results are independent of $B$ in this regime.

At each strain step, after the system is quenched at the target pressure, we measure the stress tensor elements, as defined
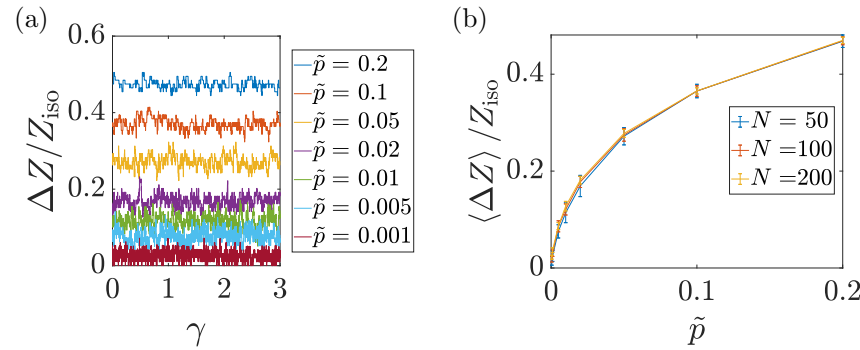

FIG. 2. (a) Excess contacts $\Delta Z / Z_{\text {iso }}$ vs the shear strain $\gamma$ for individual simulations with $N=50$, where $\Delta Z \equiv Z-Z_{\text {iso }}, Z$ is the coordination number once rattlers have been removed, and $Z_{\text {iso }}$ is the coordination number for an isostatic system. (b) Mean $\Delta Z / Z_{\text {iso }}$ vs $\tilde{p}$ over 50 simulations for $N=50,100$, and 200, showing that $\tilde{p}$ gives the fraction of extra contacts, independent of system size.

in Eq. (1), focusing on $\mu=-\sigma_{x y} / p$, as shown in Fig. 1(c). We measure $\mu$ from $0 \leqslant \gamma \leqslant 3$ in increments of $\Delta \gamma=10^{-4}$ for a total of 30001 states per simulation. For each value of $N$ and $\tilde{p}$, we simulate an ensemble of 400 systems.

We quantify distance above isostaticity by $\tilde{p}=p / K$, which gives an estimate of the relative overlap between particles (i.e., $\tilde{p}=0.01$ corresponds to particle-particle overlaps of roughly $0.01 D$ ). Figure $1(\mathrm{a})$ and 1 (b) show $\tilde{p}=0.2$ and $\tilde{p}=0.001$, respectively, with $N=24$. Overcompression yields excess contacts such that $\Delta Z / Z_{\text {iso }} \sim \sqrt{\tilde{p}}$, where $\Delta Z \equiv\left(Z-Z_{\text {iso }}\right), Z$ is the number of contacts per particle, $Z_{\text {iso }} \equiv 2\left[d\left(N-N_{r}\right)-\right.$ $d+1] /\left(N-N_{r}\right)$ is the isostatic number of contacts, $d$ is the number of spatial dimensions, and $N_{r}$ is the number of rattlers [4,33-35]. Figure 2(a) shows $\Delta Z / Z_{\text {iso }}$ plotted versus $\gamma$ for $N=50$ and varying $\tilde{p}$. These curves fluctuate around a fixed value but show no trend, indicating that the shearing does not change $Z$ on average. Figure 2(b) shows that the average value $\langle\Delta Z\rangle / Z_{\text {iso }}$ vs $\tilde{p}$ is similar for $N=50,100$, and 200. Thus, the fraction of excess contacts, a measure of distance to isostaticity, is set by $\tilde{p}$ and is nearly independent of system size [36] or the presence of shear deformation [37].

Scaling near yield. As shown in Fig. 1(c), $\mu$ increases with $\gamma$ and then plateaus as potential energy is released in intermittent slips [38-44]. This curve represents a series of jammed states that the system passes through while sheared. The fluctuations in $\mu$ decrease with increasing $N$ for all $\tilde{p}$, and we exploit the size scaling in these fluctuations (as in Ref. [45]) to demonstrate and quantify diverging spatial correlations near $\mu_{c}$. Most importantly, we show that this scaling description is nearly independent of the distance to isostaticity.

To accomplish this, we use finite size scaling on three quantities for each $\mu$ and $N$ : (1) the cumulative distribution function $F$ of states above a particular value of $\mu$ during the slip avalanche regime, defined as $\gamma>0.5$ (our results are insensitive to this choice); (2) the shear strain $\gamma_{\text {slip }}$ between mechanically stable (MS) states with an internal shear stress of at least $\mu$; and (3) the shear strain $\gamma_{\mathrm{ms}}$ required to find the first MS state with an internal shear stress of at least $\mu$. Figure $1(\mathrm{c})$ depicts $\gamma_{\mathrm{ms}}$ and $\gamma_{\text {slip }}$ for a given $\mu(\gamma)$ curve. Figures 3(a)-3(c) show these quantities plotted as functions of $\mu$ or $N$, where ensemble averages are denoted with angle brackets. The data shown in Fig. 3 represent only a single value of $\tilde{p}=0.05$ in $2 \mathrm{D}$, but it is typical of all $\tilde{p}$ in both $2 \mathrm{D}$ 


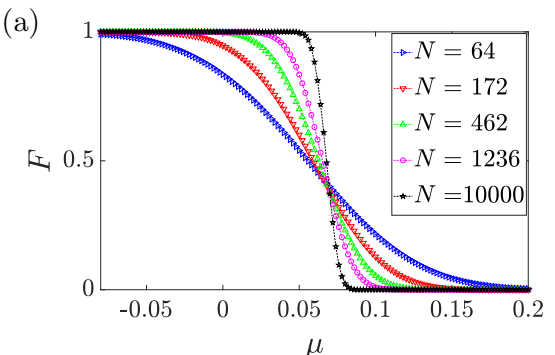

(d)

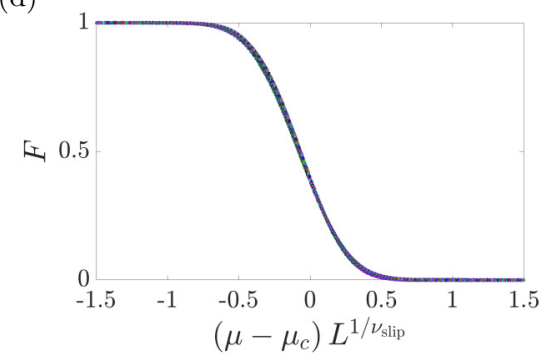

(b)

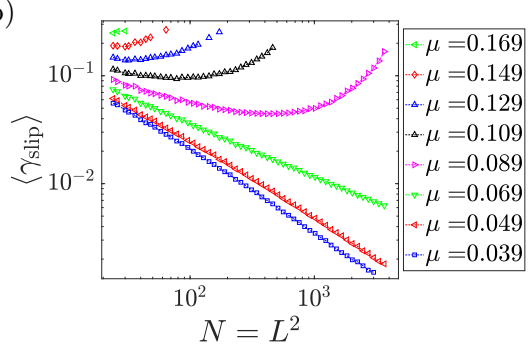

(e)

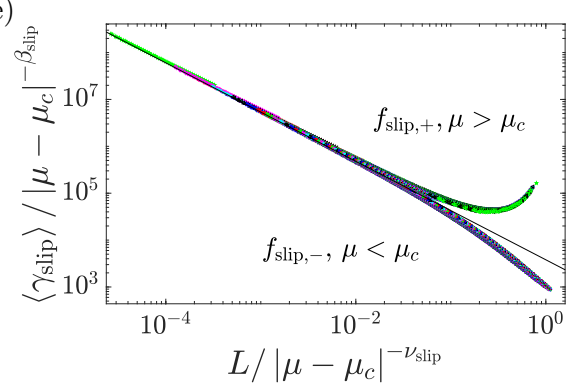

(c)

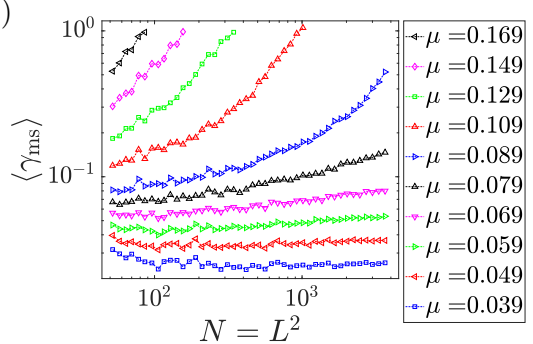

(f)

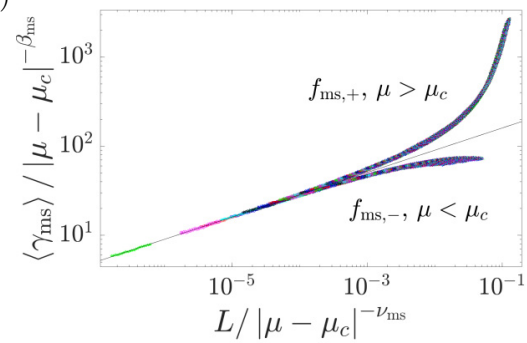

FIG. 3. Scaling collapses in 2D at high dimensionless pressure $\tilde{p}=0.05$. Fraction of states $F$ above $\mu$, unscaled (a) and scaled (d), with $\mu_{c}=0.061$ and $v_{\text {slip }}=1.09$. $\left\langle\gamma_{\text {slip }}\right\rangle$ vs $N$, unscaled (b) and scaled (e), for $24 \leqslant N \leqslant 3654$, with $\mu_{c}=0.061, v_{\text {slip }}=1.09$, and $\beta_{\text {slip }} / v_{\text {slip }}=$ -1.05. (c), (f) Mean strain $\left\langle\gamma_{\mathrm{ms}}\right\rangle$, unscaled (c) and scaled (f), to the first mechanically stable (MS) state at dimensionless shear stress $\mu$, for $24 \leqslant N \leqslant 3654$, collapsed onto the proposed scaling form with $\mu_{c}=0.061, v_{\mathrm{ms}}=1.8$, and $\beta_{\mathrm{ms}} / v_{\mathrm{ms}}=0.25$. In all three cases, the scaled data include all values of $\mu$ and $N$, and the unscaled plots show only selected curves. The data for $F$ are plotted vs $\mu$ with different curves representing different $N$, and $\left\langle\gamma_{\text {slip }}\right\rangle$ and $\left\langle\gamma_{\mathrm{ms}}\right\rangle$ are plotted vs system size with different curves representing different values of $\mu$.

and 3D, as we demonstrate below in Figs. 4 and 5. As $N$ is increased, the fluctuations in $\mu$ decrease, and $F$ approaches a step function, as shown in Fig. 3(a). Thus, MS states vanish sharply at some value $\mu=\mu_{c}(\tilde{p})$ in the large system limit. Figure 3(b) shows $\left\langle\gamma_{\text {slip }}\right\rangle$, where we require at least one $\gamma_{\text {slip }}$ measurement per simulation. Our results are insensitive to this choice, unless the number of samples becomes very small. For $\mu<\mu_{c},\left\langle\gamma_{\text {slip }}\right\rangle$ monotonically decreases with increasing $N$. For $\mu>\mu_{c},\left\langle\gamma_{\text {slip }}\right\rangle$ first decreases and then increases with increasing $N$. Finally, $\left\langle\gamma_{\mathrm{ms}}\right\rangle$ is nearly independent of $N$ for small $\mu$ and increases strongly with $N$ for larger $\mu$.

To collapse these curves, we posit a diverging length scale $\xi \propto\left|\mu-\mu_{c}\right|^{-v}$. In this case, finite size effects should enter through the quantity $L / \xi$, where $L \equiv N^{1 / d}$ with $d$ being the number of spatial dimensions. An equivalent scaling can also be written using $\left(\mu-\mu_{c}\right) L^{1 / \nu}$; see Refs. [27,32] for further discussions on similar systems. Figures 3(d)-3(f) show that the data in Figs. 3(a)-3(c) collapse according to

$$
\begin{aligned}
F & =f\left(\left(\mu-\mu_{c}\right) L^{1 / v_{\text {slip }}}\right), \\
\left\langle\gamma_{\text {slip }}\right\rangle & =\left|\mu-\mu_{c}\right|^{-\beta_{\text {slip }}} f_{\text {slip }, \pm}\left(\frac{L}{\left|\mu-\mu_{c}\right|^{-v_{\text {slip }}}}\right), \\
\left\langle\gamma_{\mathrm{ms}}\right\rangle & =\left|\mu-\mu_{c}\right|^{-\beta_{\mathrm{ms}}} f_{\mathrm{ms}, \pm}\left(\frac{L}{\left|\mu-\mu_{c}\right|^{-v_{\mathrm{ms}}}}\right) .
\end{aligned}
$$

Here, $f_{\mathrm{ms}, \pm}$ and $f_{\text {slip }, \pm}$ are dual-branch functions, with + and - denoting $\mu$ above or below $\mu_{c}$, respectively. Interestingly, we require two distinct values of $\nu$. For the initial strain, we find $v_{\mathrm{ms}} \approx 1.8$, while in the slip avalanche regime, we find $v_{\text {slip }} \approx 1.1$ in $2 \mathrm{D}$ and $v_{\text {slip }} \approx 0.8$ in $3 \mathrm{D}$. The value $v_{\text {slip }} \approx$ 1.8 agrees with our previous result [32], which was only calculated near to isostaticity; in Fig. 3, it is calculated far from isostaticity. The difference between $v_{\text {slip }}$ and $v_{\mathrm{ms}}$ suggests that there are important differences in how MS states are accessed between these two regimes. We obtain the critical parameters $\mu_{c}$ and $\nu_{\text {slip }}$ by fitting the collapsed curves to appropriate functional forms using a Levenberg-Marquardt method [27,32]. We exclude system sizes with $N<N_{\min }$, and vary $N_{\text {min }}$ until our fits become insensitive to our choice of $N_{\text {min }}$. We also performed the corrections-to-scaling analysis

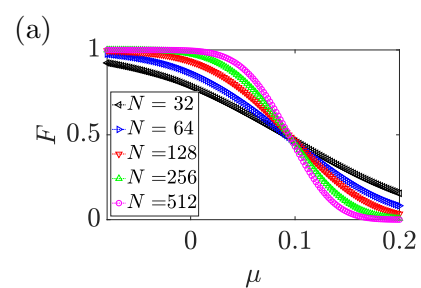

(c)

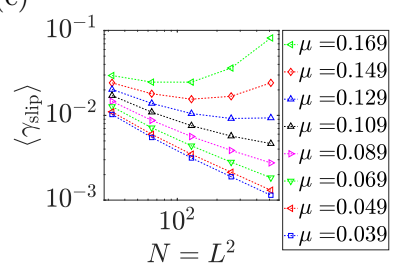

(b)

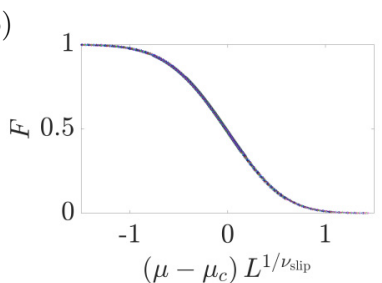

(d)

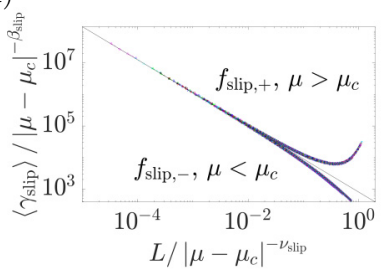

FIG. 4. Scaling collapses in 2D at low dimensionless pressure $\tilde{p}=0.0005,32 \leqslant N \leqslant 512$. Fraction of states $F$ above $\mu$, unscaled (a) and scaled (b), with $\mu_{c}=0.097$ and $v_{\text {slip }}=1.10 .\left\langle\gamma_{\text {slip }}\right\rangle$ vs $N$, unscaled (c) and scaled (d), with $\mu_{c}=0.097, v_{\text {slip }}=1.10$, and $\beta_{\text {slip }} / v_{\text {slip }}=-1.05$. In both cases, the unscaled plots show selected values of $N$ or $\mu$ while the scaled plots show all data. 


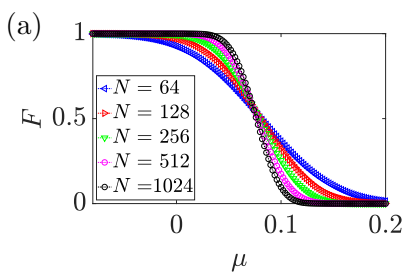

(c)

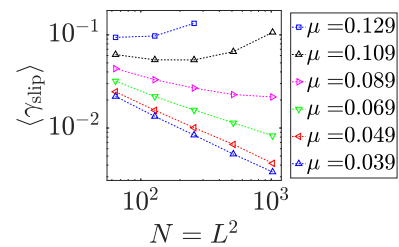

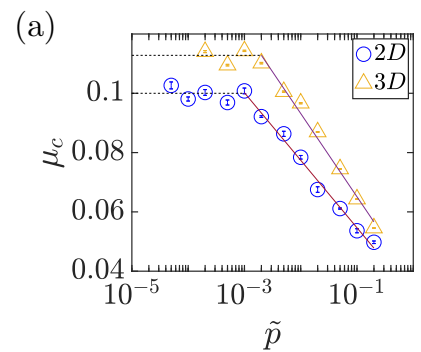

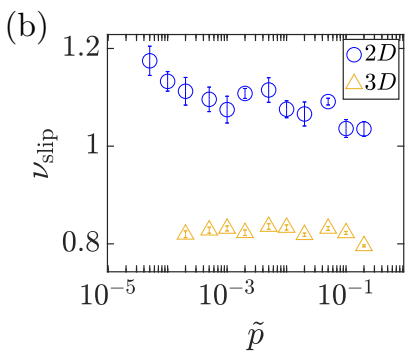

(d)

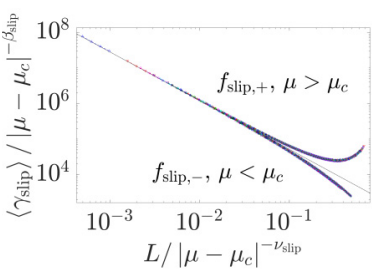

FIG. 5. Scaling collapses in 3D at high dimensionless pressure $\tilde{p}=0.05,64 \leqslant N \leqslant 1024$. Fraction of states $F$ above $\mu$, unscaled (a) and scaled (b), with $\mu_{c}=0.074$ and $v_{\text {slip }}=0.832 .\left\langle\gamma_{\text {slip }}\right\rangle$ vs $N$, unscaled (c) and scaled (d), with $\mu_{c}=0.074, v_{\text {slip }}=0.832$, and $\beta_{\text {slip }} / v_{\text {slip }}=-1.37$. In both cases, the unscaled plots show selected values of $N$ or $\mu$ while the scaled plots show all data.

in Ref. [46], which yields the same result we find with the scaling forms in Eqs. (2)-(4).

We then perform the same analysis for varying $\tilde{p}$ over the ranges $5 \times 10^{-5} \leqslant \tilde{p} \leqslant 2 \times 10^{-1}$ in $2 \mathrm{D}$ and $2 \times 10^{-4} \leqslant \tilde{p} \leqslant$ $2 \times 10^{-1}$ in $3 \mathrm{D}$, spanning from near isostaticity (where $\xi_{J}$ is large) to far from isostaticity (where $\xi_{J}$ is small). The scaling description in Eqs. (2)-(4) and shown in Fig. 3 holds for all values of $\tilde{p}$ in both $2 \mathrm{D}$ and $3 \mathrm{D}$. We show data for an additional pressure in $2 \mathrm{D}, \tilde{p}=0.0005$, in Fig. 4 . We also show data in $3 \mathrm{D}$ with $\tilde{p}=0.05$ in Fig. 5 . In both cases, the scaling functions are almost indistinguishable from those shown in Fig. 3. Figure 6 shows the critical parameters $\mu_{c}$ and $v_{\text {slip }}$ plotted as a function of $\tilde{p}$. Each data point in Fig. 6 represents a fit of all data (as in Figs. 3-5) over many system sizes (typically $32 \leqslant N \leqslant 1024$ ) with 400 simulations per system size, so the plateau in Fig. 6 is not a system size effect. We find that $\mu_{c}$ is independent of $\tilde{p}$ for $\tilde{p} \leqslant 10^{-3}$ and decreases logarithmically for $\tilde{p}>10^{-3}$, which agrees with Favier de Coulomb et al. [37]. This occurs as excess contacts are added, which changes the structure of the force networks.

However, we observe no similar crossover behavior as the distance to isostaticity is varied in any other aspects of the scaling behavior. The critical exponents, as shown in Fig. 6(b), and the scaling functions, as shown in Figs. 3-5, are highly insensitive to $\tilde{p}$, despite the wide variation in distance to isostaticity. Specifically, we find $v_{\text {slip }} \approx 1.1 \pm 0.1$ in $2 \mathrm{D}, v_{\text {slip }} \approx 0.8 \pm 0.03$ in $3 \mathrm{D}, \beta_{\text {slip }} / v_{\text {slip }} \approx-1 \pm 0.1$ in $2 \mathrm{D}$, and $\beta_{\text {slip }} / \nu_{\text {slip }} \approx-1.3 \pm 0.1$ in $3 \mathrm{D}$. The uncertainty is estimated from the scatter in the data for different $\tilde{p}$, as seen in Fig. 6. For the initial shear regime, we find that $\nu_{\mathrm{ms}} \approx 1.8$ is insensitive to $\tilde{p}$. However, $\beta_{\mathrm{ms}} / \nu_{\mathrm{ms}}$ appears to vary from roughly 0.2 at high $\tilde{p}$ to 0.6 at low $\tilde{p}$. This again points to potentially important differences between how MS states are explored between the slip avalanche and initial shear regimes and may have consequences for size-dependent arrest transitions $[32,47,48]$.

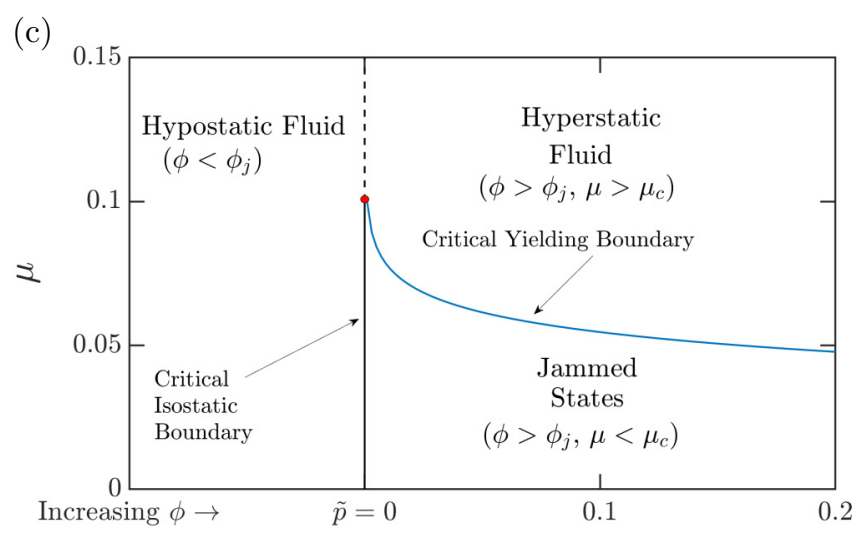

FIG. 6. (a), (b) Values for (a) $\mu_{c}$ and (b) $v_{\text {slip }}$ vs $\tilde{p}$, measured using the fitting protocol described in the text. Results in 2D and 3D are denoted by circles and triangles, respectively. Solid lines in (a) represent a linear fit of $\mu_{c}$ vs $\log \tilde{p}$ from $\tilde{p}=10^{-3}$ through $2 \times 10^{-1}$, while the dashed lines represent the large-stiffness limit. (c) Phase diagram summarizing our results. The solid blue line represents the estimation of $\mu_{c}(\tilde{p})$ in $2 \mathrm{D}$ from (a).

Discussion. We have shown here that sheared amorphous soft sphere packings display finite size scaling that is consistent with a diverging length scale $\xi \propto\left|\mu-\mu_{c}\right|^{-v}$. The value of $\mu_{c}$ varies as $\tilde{p}$ is changed and extra contacts are added, but the forms of the scaling functions (as shown in Figs. 3-5) and the values of the critical exponents are nearly independent of distance to isostaticity over nearly four orders of magnitude in $\tilde{p}$. Considering the correlation length for isostaticity $\xi_{J} \propto$ $\left|\phi-\phi_{c}\right|^{-v_{J}}$, if one assumes that $v_{J}$ is order unity [27] and $\tilde{p} \propto\left(\phi-\phi_{c}\right)$ for harmonic interactions [2], then varying $\tilde{p}$ over this range represents $\xi_{J}$ varying over a similar range. This represents an enormous variation with respect to the isostatic critical point, implying that the distance to isostaticity does not control the critical behavior we demonstrate here. Our results suggest that yielding in, e.g., emulsions, foams, or granular materials is controlled by an underlying nonequilibrium critical transition that is distinct from isostaticity. We note that $v_{\text {slip }} \approx 1.1$ for $2 \mathrm{D}$ and $v_{\text {slip }} \approx 0.8$ in $3 \mathrm{D}$ are similar to the values $v \approx 1.1$ for $2 \mathrm{D}$ and $v \approx 0.7$ for $3 \mathrm{D}$ from Ref. [45], which presented a scaling description for yielding in amorphous materials $[38,40,43]$.

Figure 6(c) shows the Liu-Nagel jamming phase diagram from, e.g., Refs. [2,49,50] and many others, but with $\tilde{p}$ on the horizontal axis and $\mu=\tau / p$ on the vertical axis. The solid blue line represents the critical yielding boundary in 2D from Fig. 6(a), and the solid vertical black line represents the isostatic critical transition. Jammed states exist only in the lower right region, above isostaticity and below the 
critical yielding boundary. Unjammed or fluidlike states can be either hypostatic $\left(Z<Z_{\text {iso }}\right.$ and $\left.p=0\right)$ or hyperstatic $\left(Z>Z_{\text {iso }}\right.$ and $\left.p>0\right)$. Some previous work on critical scaling near isostaticity has studied the onset of yield stress behavior under shear at varied $\phi[24,25,27,28,49]$. Such a system is situated at the "triple point" indicated by the red dot at the intersection of the jamming and yielding lines in Fig. 6(c). A complete theory may be able to unify these two critical transitions by a better understanding of the behavior at this point.
[1] H. M. Jaeger, S. R. Nagel, and R. P. Behringer, Granular solids, liquids, and gases, Rev. Mod. Phys. 68, 1259 (1996).

[2] C. S. O'Hern, L. E. Silbert, A. J. Liu, and S. R. Nagel, Jamming at zero temperature and zero applied stress: The epitome of disorder, Phys. Rev. E 68, 011306 (2003).

[3] A. Donev, S. Torquato, F. H. Stillinger, and R. Connelly, Jamming in hard sphere and disk packings, J. Appl. Phys. 95, 989 (2004).

[4] M. Van Hecke, Jamming of soft particles: Geometry, mechanics, scaling and isostaticity, J. Phys.: Condens. Matter. 22, 033101 (2009).

[5] A. J. Liu and S. R. Nagel, The jamming transition and the marginally jammed solid, Annu. Rev. Condens. Matter Phys. 1, 347 (2010).

[6] D. C. Drucker and W. Prager, Soil mechanics and plastic analysis or limit design, Q. Appl. Math. 10, 157 (1952).

[7] B. S. Gardiner, B. Z. Dlugogorski, G. J. Jameson, and R. P. Chhabra, Yield stress measurements of aqueous foams in the dry limit, J. Rheol. 42, 1437 (1998).

[8] M. Toiya, J. Stambaugh, and W. Losert, Transient and Oscillatory Granular Shear Flow, Phys. Rev. Lett. 93, 088001 (2004).

[9] F. da Cruz, S. Emam, M. Prochnow, J.-N. Roux, and F. Chevoir, Rheophysics of dense granular materials: Discrete simulation of plane shear flows, Phys. Rev. E 72, 021309 (2005).

[10] N. Xu and C. S. O'Hern, Measurements of the yield stress in frictionless granular systems, Phys. Rev. E 73, 061303 (2006).

[11] P. Jop, Y. Forterre, and O. Pouliquen, A constitutive law for dense granular flows, Nature (London) 441, 727 (2006).

[12] P.-E. Peyneau and J.-N. Roux, Frictionless bead packs have macroscopic friction, but no dilatancy, Phys. Rev. E 78, 011307 (2008).

[13] P.-E. Peyneau and J.-N. Roux, Solidlike behavior and anisotropy in rigid frictionless bead assemblies, Phys. Rev. E 78, 041307 (2008).

[14] J. Goyon, A. Colin, G. Ovarlez, A. Ajdari, and L. Bocquet, Spatial cooperativity in soft glassy flows, Nature (London) $\mathbf{4 5 4}$, 84 (2008).

[15] C. Masselon, J.-B. Salmon, and A. Colin, Nonlocal Effects in Flows of Wormlike Micellar Solutions, Phys. Rev. Lett. 100, 038301 (2008).

[16] L. Bocquet, A. Colin, and A. Ajdari, Kinetic Theory of Plastic Flow in Soft Glassy Materials, Phys. Rev. Lett. 103, 036001 (2009).

[17] P. Jop, V. Mansard, P. Chaudhuri, L. Bocquet, and A. Colin, Microscale Rheology of a Soft Glassy Material Close to Yielding, Phys. Rev. Lett. 108, 148301 (2012).

[18] K. Kamrin and G. Koval, Nonlocal Constitutive Relation for Steady Granular Flow, Phys. Rev. Lett. 108, 178301 (2012).

[19] D. L. Henann and K. Kamrin, A predictive, size-dependent continuum model for dense granular flows, Proc. Natl. Acad. Sci. U.S.A. 110, 6730 (2013).
[20] M. Bouzid, M. Trulsson, P. Claudin, E. Clément, and B. Andreotti, Nonlocal Rheology of Granular Flows Across Yield Conditions, Phys. Rev. Lett. 111, 238301 (2013).

[21] M. Bouzid, A. Izzet, M. Trulsson, E. Clément, P. Claudin, and B. Andreotti, Non-local rheology in dense granular flows, Eur. Phys. J. E 38, 125 (2015).

[22] J. A. Drocco, M. B. Hastings, C. J. O. Reichhardt, and C. Reichhardt, Multiscaling at Point $J$ : Jamming is a Critical Phenomenon, Phys. Rev. Lett. 95, 088001 (2005).

[23] W. G. Ellenbroek, E. Somfai, M. van Hecke, and W. van Saarloos, Critical Scaling in Linear Response of Frictionless Granular Packings Near Jamming, Phys. Rev. Lett. 97, 258001 (2006).

[24] P. Olsson and S. Teitel, Critical Scaling of Shear Viscosity at the Jamming Transition, Phys. Rev. Lett. 99, 178001 (2007).

[25] K. N. Nordstrom, E. Verneuil, P. E. Arratia, A. Basu, Z. Zhang, A. G. Yodh, J. P. Gollub, and D. J. Durian, Microfluidic Rheology of Soft Colloids Above and Below Jamming, Phys. Rev. Lett. 105, 175701 (2010).

[26] B. P. Tighe, E. Woldhuis, J. J. C. Remmers, W. van Saarloos, and M. van Hecke, Model for the Scaling of Stresses and Fluctuations in Flows Near Jamming, Phys. Rev. Lett. 105, 088303 (2010).

[27] P. Olsson and S. Teitel, Critical scaling of shearing rheology at the jamming transition of soft-core frictionless disks, Phys. Rev. E 83, 030302(R) (2011).

[28] J. Paredes, M. A. J. Michels, and D. Bonn, Rheology Across the Zero-Temperature Jamming Transition, Phys. Rev. Lett. 111, 015701 (2013).

[29] C. P. Goodrich, A. J. Liu, and J. P. Sethna, Scaling ansatz for the jamming transition, Proc. Natl. Acad. Sci. U.S.A. 113, 9745 (2016).

[30] L. E. Silbert, A. J. Liu, and S. R. Nagel, Vibrations and Diverging Length Scales Near the Unjamming Transition, Phys. Rev. Lett. 95, 098301 (2005).

[31] M. Wyart, L. E. Silbert, S. R. Nagel, and T. A. Witten, Effects of compression on the vibrational modes of marginally jammed solids, Phys. Rev. E 72, 051306 (2005).

[32] A. H. Clark, J. D. Thompson, M. D. Shattuck, N. T. Ouellette, and C. S. O'Hern, Critical scaling near the yielding transition in granular media, Phys. Rev. E 97, 062901 (2018).

[33] S. Papanikolaou, C. S. O'Hern, and M. D. Shattuck, Isostaticity at Frictional Jamming, Phys. Rev. Lett. 110, 198002 (2013).

[34] T. Shen, S. Papanikolaou, C. S. O'Hern, and M. D. Shattuck, Statistics of Frictional Families, Phys. Rev. Lett. 113, 128302 (2014).

[35] K. VanderWerf, W. Jin, M. D. Shattuck, and C. S. O’Hern, Hypostatic jammed packings of frictionless nonspherical particles, Phys. Rev. E 97, 012909 (2018).

[36] C. P. Goodrich, A. J. Liu, and S. R. Nagel, Finite-Size Scaling at the Jamming Transition, Phys. Rev. Lett. 109, 095704 (2012). 
[37] A. Favier de Coulomb, M. Bouzid, P. Claudin, E. Clément, and B. Andreotti, Rheology of granular flows across the transition from soft to rigid particles, Phys. Rev. Fluids 2, 102301 (2017).

[38] M. C. Miguel, A. Vespignani, S. Zapperi, J. Weiss, and J.-R. Grasso, Intermittent dislocation flow in viscoplastic deformation, Nature (London) 410, 667 (2001).

[39] F. Dalton and D. Corcoran, Self-organized criticality in a sheared granular stick-slip system, Phys. Rev. E 63, 061312 (2001).

[40] C. E. Maloney and A. Lemaître, Amorphous systems in athermal, quasistatic shear, Phys. Rev. E 74, 016118 (2006).

[41] K. E. Daniels and N. W. Hayman, Force chains in seismogenic faults visualized with photoelastic granular shear experiments, J. Geophys. Res.: Solid Earth 113, B11411 (2008).

[42] K. A. Dahmen, Y. Ben-Zion, and J. T. Uhl, A simple analytic theory for the statistics of avalanches in sheared granular materials, Nat. Phys. 7, 554 (2011).

[43] K. M. Salerno, C. E. Maloney, and M. O. Robbins, Avalanches in Strained Amorphous Solids: Does Inertia Destroy Critical Behavior? Phys. Rev. Lett. 109, 105703 (2012).
[44] J. Barés, D. Wang, D. Wang, T. Bertrand, C. S. O’Hern, and R. P. Behringer, Local and global avalanches in a twodimensional sheared granular medium, Phys. Rev. E 96, 052902 (2017).

[45] J. Lin, E. Lerner, A. Rosso, and M. Wyart, Scaling description of the yielding transition in soft amorphous solids at zero temperature, Proc. Natl. Acad. Sci. U.S.A. 111, 14382 (2014).

[46] D. Vågberg, D. Valdez-Balderas, M. A. Moore, P. Olsson, and S. Teitel, Finite-size scaling at the jamming transition: Corrections to scaling and the correlation-length critical exponent, Phys. Rev. E 83, 030303(R) (2011).

[47] K. Kamrin and D. L. Henann, Nonlocal modeling of granular flows down inclines, Soft Matter 11, 179 (2015).

[48] I. Srivastava, L. E. Silbert, G. S. Grest, and J. B. Lechman, Flow Arrest Transitions in Frictional Granular Matter, Phys. Rev. Lett. 122, 048003 (2019).

[49] C. Heussinger and J.-L. Barrat, Jamming Transition as Probed by Quasistatic Shear Flow, Phys. Rev. Lett. 102, 218303 (2009).

[50] D. Bi, J. Zhang, B. Chakraborty, and R. P. Behringer, Jamming by shear, Nature (London) 480, 355 (2011). 